\title{
Diffusion Tensor MRI to Assess Damage in Healthy and Dystrophic Skeletal Muscle after Lengthening Contractions
}

\author{
Alan B. McMillan, ${ }^{1}$ Da Shi, ${ }^{1}$ Stephen J. P. Pratt, ${ }^{2}$ and Richard M. Lovering ${ }^{2}$ \\ ${ }^{1}$ Department of Diagnostic Radiology, University of Maryland School of Medicine, Baltimore, MD 21201, USA \\ ${ }^{2}$ Department of Orthopaedics, University of Maryland School of Medicine, Baltimore, MD 21201, USA
}

Correspondence should be addressed to Richard M. Lovering, rlovering@som.umaryland.edu

Received 13 May 2011; Revised 2 July 2011; Accepted 4 August 2011

Academic Editor: Robert J. Bloch

Copyright (C) 2011 Alan B. McMillan et al. This is an open access article distributed under the Creative Commons Attribution License, which permits unrestricted use, distribution, and reproduction in any medium, provided the original work is properly cited.

\begin{abstract}
The purpose of this study was to determine if variables calculated from diffusion tensor imaging (DTI) would serve as a reliable marker of damage after a muscle strain injury in dystrophic $(m d x)$ and wild type (WT) mice. Unilateral injury to the tibialis anterior muscle (TA) was induced in vivo by 10 maximal lengthening contractions. High resolution T1- and T2-weighted structural MRI, including T2 mapping and spin echo DTI was acquired on a 7T small animal MRI system. Injury was confirmed by a significant loss of isometric torque ( $85 \%$ in $m d x$ versus $42 \%$ in WT). Greater increases in apparent diffusion coefficient (ADC), axial, and radial diffusivity ( $\mathrm{AD}$ and $\mathrm{RD}$ ) of the injured muscle were present in the $m d x$ mice versus controls. These changes were paralleled by decreases in fractional anisotropy (FA). Additionally, T2 was increased in the $m d x$ mice, but the spatial extent of the changes was less than those in the DTI parameters. The data suggest that DTI is an accurate indicator of muscle injury, even at early time points where the MR signal changes are dominated by local edema.
\end{abstract}

\section{Introduction}

The muscular dystrophies (MDs) are a heterogeneous group of inherited disorders characterized by progressive weakness and degeneration of skeletal muscles. Duchenne muscular dystrophy (DMD), the most common form of MD, is an Xlinked disorder that was first described over a century ago [1]. DMD is caused by the absence of dystrophin, a $427 \mathrm{kDa}$ protein found on the cytoplasmic surface of the plasma membrane of muscle fibers (the sarcolemma) in skeletal and cardiac muscle. It is well established that the absence of dystrophin leads to the impaired linkage between the cytoskeleton and the extracellular matrix protein laminin, which is thought to result in fragility of the sarcolemma and a disruption in the proper transmission of force during contraction.

Muscle strains are one of the most common complaints treated by physicians [2]. When an activated muscle lengthens because the external load exceeds the tension generated by the muscle contraction, this is termed a lengthening ("eccentric") contraction. The force generated during a max- imal lengthening contraction is at least twofold the force developed during a maximal isometric contraction; therefore lengthening contractions are more likely to produce damage than either isometric or concentric contractions [3]. Eccentric contractions are especially harmful to dystrophic muscle $[4,5]$.

Plain films, or X-rays, are not very useful for imaging muscle pathology, unless heterotopic bone formation has occurred within the muscle. Diagnosis of acute muscle strains is still typically made based on physical examination and patient history, but muscle injuries can be detected with MR imaging methods [6]. As noninvasive technology continues to improve and imaging, such as magnetic resonance imaging (MRI), becomes more commonplace, these tools will play a greater role in diagnosis, prognosis, and in rehabilitation planning [7]. Unlike X-rays, MRI offers superb tissue contrast and has high sensitivity to the hemorrhage and edema that follow muscle injuries. This, together with the capability to evaluate multiple arbitrary anatomic planes, make it the ideal technique to evaluate muscle injures. Because of the similarities between muscle injury and muscle 
disease, MRI may yield useful information for both of these conditions.

Conventionally, muscle strains are revealed best by T2weighted MRI images, which optimize contrast between injured muscles with edema (increased signal intensity) and normal uninjured muscles. More recently, diffusion tensor imaging (DTI) has been explored as a more accurate marker for muscle damage compared to T2-weighted MRI [8]. The variables obtained with DTI, at least in other tissues such as the brain [9], show a strong and rapid response to damage, whereas the T2 signal can take a prolonged period to change. DTI is based on measurement of the apparent diffusion of water in tissues. Self-diffusion of water in tissue is restricted by membranes, resulting in an apparent diffusion coefficient (ADC) that is lower than the free diffusion coefficient and is orientation dependent for elongated structures. In short, DTI can be used to determine the three-dimensional architecture of skeletal muscle $[10,11]$ as well as provide useful information regarding muscle damage [12].

The purpose of this study was to determine if measurements obtained from DTI would serve as a reliable marker of damage after a muscle strain injury in dystrophic $(m d x)$ and control mice. We hypothesize that DTI biomarkers would provide a more informative assessment of muscle injury than T2 and that these measures will further elucidate the increased susceptibility to injury of the $m d x$ model.

\section{Materials and Methods}

2.1. Injury. All protocols were approved by the University of Maryland Institutional Animal Care \& Use Committee (IACUC). The injury model results in a significant and reproducible injury and has been described in detail previously [13-16]. Briefly, 5 adult healthy (C57BL/10ScSn) and 5 dystrophic $\left(\mathrm{C} 57 \mathrm{BLScSn}-\mathrm{DMD}^{m d x}\right.$ ) male mice were purchased (The Jackson Laboratory, Bar Harbor, Me) and were approximately 8 weeks old at the start of experiments. Before each injury protocol, mice were anesthetized ( $4-5 \%$ isoflurane in an induction chamber, then $\sim 2 \%$ isoflurane via a nosecone for maintenance) using a precision vaporizer (cat no. 91103, Vet Equip, Inc, Pleasanton, Calif). Sterile ophthalmic cream (Paralube Vet Ointment, PharmaDerm, Floham Park, NJ) was applied to each eye to protect the corneas from drying. During the procedure, the animal was kept warm by use of a heat lamp.

With the animal supine, the hindlimb was stabilized, and the foot was secured onto a plate, the axis of which was attached to a stepper motor (model T8904, NMB Technologies, Chatsworth, Calif) and a torque sensor (QWFK$8 \mathrm{M}$, Sensotec) to measure torque. The fibular nerve was stimulated via subcutaneous needle electrodes (J05 Needle Electrode Needles, 36BTP, Jari Electrode Supply, Gilroy, Calif), and proper electrode position was determined by a series of isometric twitches. Impulses generated by an S48 square pulse stimulator (Grass Instruments, West Warwick, RI) were $1 \mathrm{~ms}$ in duration and passed through a PSIU6 stimulator isolation unit (Grass Instruments, West Warwick, RI). In addition to visual confirmation of isolated dorsiflexion, an increase in twitch torque in response to increasing voltage indicated that opposing plantarflexor muscles were not being simultaneously stimulated [17].

The majority of torque produced by the dorsiflexors is from the tibialis anterior muscle (TA) [18], and we have shown previously that this model results in injury to this muscle $[15,16,19,20]$. A custom program based on commercial software (Labview version 8.5, National Instruments, Austin, Tex) was used to synchronize contractile activation and the onset of ankle rotation. Injury resulted from 10 forced lengthening (plantarflexion) contractions through a $0^{\circ}-70^{\circ}$ arc of motion (with the foot orthogonal to the tibia, considered $0^{\circ}$ ). A sham procedure was performed whereby the identical protocol was performed (including stimulation), but without lengthening.

2.2. Outcome Measures. A maximal isometric contraction (200 ms duration) of the dorsiflexors was used to measure maximal torque before injury. For each animal, maximal isometric torque was also measured 5 minutes after injury (to measure force lost due to injury). All isometric contractions were performed with the ankle at $20^{\circ}$ into plantarflexion, a position that results in optimal force production.

2.3. In Vivo Imaging. High resolution MRI (magnetic resonance imaging) was performed on a Bruker Biospin (Billerica, Mass) 7.0 Tesla MR system equipped with a $12 \mathrm{~cm}$ gradient insert $(660 \mathrm{mT} / \mathrm{m}$ maximum gradient, $4570 \mathrm{~T} / \mathrm{m} / \mathrm{s}$ maximum slew rate) running Paravision 5.0 software to assess muscle damage on the day of injury. An MR-compatible small-animal monitoring and gating system (SA Instruments, Inc.) was used to monitor respiration rate and body temperature. Body temperature was maintained at 36$37^{\circ} \mathrm{C}$ using a warm water circulator. A custom-made holder was used to position the mouse in the supine position with both legs parallel to the bore of the magnet from knee to foot. Two hours after injury [21], animals were placed inside the scanner, with a four-channel receive-only surface coil placed anterior to the TA. Anesthesia was maintained using $2 \%$ isofluorane and was adjusted to maintain a safe respiration rate.

After imaging localizers, the following MR scans were performed: T1-weighted rapid acquisition with relaxation enhancement (RARE) with the following parameters: TE = $9.52 \mathrm{~ms}, \mathrm{TR}=1800$, echo train length $=4, \mathrm{NA}=8$, in-plane resolution $100 \times 100 \mu \mathrm{m}$, and slice thickness $=750 \mu \mathrm{m}$; dual echo PD/T2 RARE: $\mathrm{TE}=19.0 / 57.1 \mathrm{~ms}, \mathrm{TR}=5000 \mathrm{~ms}$, echo train length $=4, \mathrm{NA}=1$, in-plane resolution $100 \times 100 \mu \mathrm{m}$, and slice thickness $=750 \mu \mathrm{m}$. Spin echo (SE) diffusion tensor image data was acquired using 12 noncolinear directions: $b$ value $=350 \mathrm{~s} / \mathrm{mm}^{-2}, \mathrm{TE}=26 \mathrm{~ms}, \mathrm{TR}=4500 \mathrm{~ms}, \mathrm{NA}=1$, inplane resolution $150 \times 150 \mu \mathrm{m}$, and slice thickness $=750 \mu \mathrm{m}$. Multislice multiecho (MSME) T2 mapping image data was acquired in the same slice positions as the DTI data using $16 \mathrm{TEs}=11.4 \mathrm{~ms}$ to $182.5 \mathrm{~ms}$ with $\Delta \mathrm{TE}=11.4 \mathrm{~ms}$, $\mathrm{TR}=$ $10000 \mathrm{~ms}, \mathrm{NA}=1$, in-plane resolution $150 \times 150 \mu \mathrm{m}$, and slice thickness $=750 \mu \mathrm{m}$.

T2 mapping was performed using custom software written in MATLAB (The Mathworks; Natick, Mass) using 
nonlinear least squares to fit the measured data at each pixel to the canonical T2 signal equation. Diffusion tensor reconstruction and tractography was performed using TrackVis (Martinos Center for Biomedical Imaging; Massachusetts General Hospital; Boston, Mass) and MATLAB to calculate mean diffusivity (MD), fractional anisotropy (FA), radial diffusivity $(\mathrm{RD})$, and axial diffusivity $(\mathrm{AD})$ images. A region of interest (ROI) in the TA and tractography was used to guide bilateral segmentation of the TA. Diffusion tensor tractography was calculated using the fiber assignment by continuous tracking (FACT) method [22] with a termination criteria of an angle greater than $35^{\circ}$, followed by subsequent spline filtering. Tracts were restricted to those traveling through several transverse slices of manually traced regions of interest within each of the left and right TAs. This was used to create an image mask for each muscle, which was then divided into proximal, middle, and distal sections of approximately equal length. Finally, the masks were used to calculate average measurements of $\mathrm{MD}, \mathrm{FA}, \mathrm{RD}, \mathrm{AD}$, and $\mathrm{T} 2$ within each section of the injured and uninjured muscle. These measurements were compared between normal and dystrophic mice for both the uninjured and the injured side using a Wilcoxon Rank Sum test.

2.4. Histology. After MR and functional data were collected, anesthetized animals were fixed with $4 \%$ paraformaldehyde via perfusion through the left ventricle to preserve tissue morphology. TAs were harvested, weighed, snap frozen in liquid nitrogen, and then stored at $-80^{\circ} \mathrm{C}$. Animals not perfused fixed were euthanized by carbon dioxide inhalation followed by decapitation. To study the integrity of the muscle fiber membrane, additional mice $(n=3$ each group) received an intraperitoneal injection of $1 \%$ Evans blue dye (EBD; Sigma, St. Louis, Mo) (wt/vol) in phosphatebuffered saline (PBS, $\mathrm{pH}$ 7.4) at a volume of $1 \%$ body mass (BM) (1 mg EBD/0.1 mL PBS/10 g BM). This solution was sterilized by passage through a Millex-GP $0.22 \mu \mathrm{m}$ filter (Millipore, Bedford, Mass) and administered $24 \mathrm{~h}$ before death of the animal to assure a good signal [23]. EBD binds to albumin and is detected by fluorescence microscopy (at $568 \mathrm{~nm}$ ) in the extracellular space. Presence of the proteinbound dye inside the muscle fiber indicates damage to the sarcolemma. Here, the sections were assessed blindly, and the myofibers were judged in a binary fashion, as positive or negative for intracellular EBD.

Transverse sections were cut on a cryostat $(10 \mu \mathrm{m}$ thickness) and collected onto glass slides (Superfrost Plus; VWR, West Chester, Pa). For EBD-injected tissue, myofibers with dye-labeled sarcoplasm were quantified in cross-sections under fluorescence optics [16, 24]. At least 400 fibers in 10 optical fields were assessed in 3 mice; results are expressed as percent labeled fibers. In sections from muscles without EBD, sections were stained with hematoxylin and eosin (H\&E) for tissue evaluation. Sections were randomized and viewed at 100x magnification in a Zeiss Axioskop light microscope, and pictures were taken with a digital camera (AxioCam HR using AxioVision 3.0). Each optical field contained an

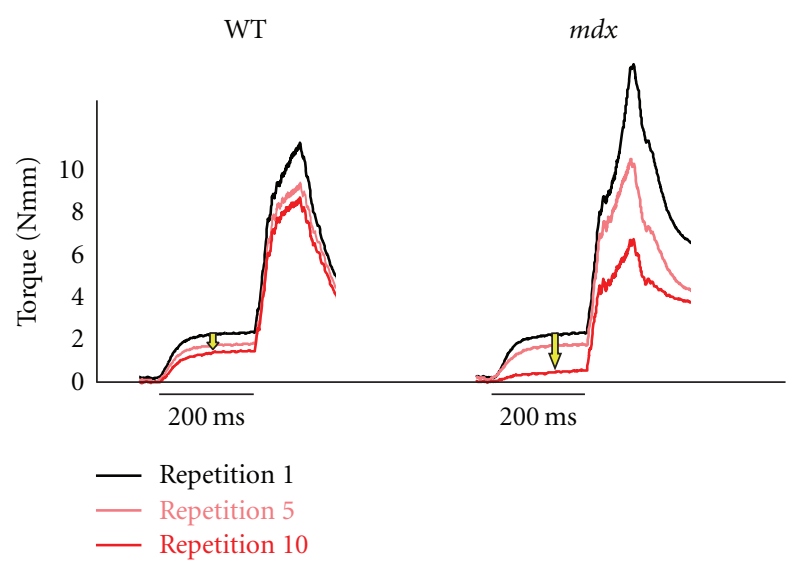

(a)

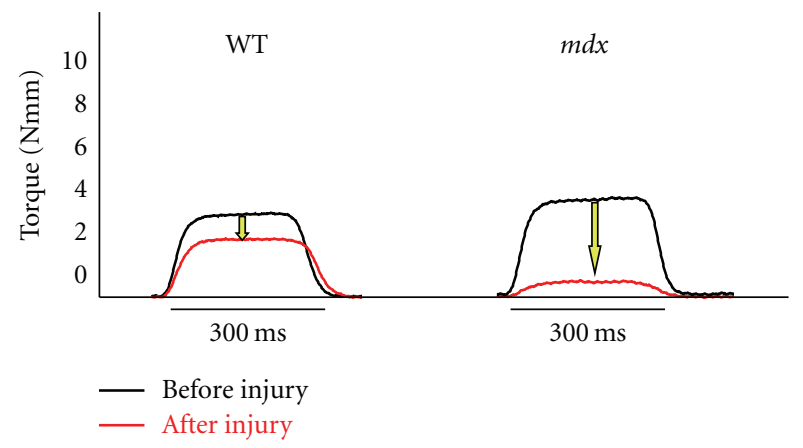

(b)

Figure 1: Mice lacking dystrophin are more susceptible to injury. Producing the injury, the fibular (aka peroneal) nerve was used to stimulate the dorsiflexor muscles supramaximally while moving the plate forced the foot into plantar flexion. Injury was induced by 10 large strain lengthening ("eccentric") contractions through a $70^{\circ}$ arc of motion. Maximal isometric torque was measured before and after injury in wild type (WT) and mice lacking dystrophin $(m d x)$. The dorsiflexors were maximally activated isometrically for $200 \mathrm{~ms}$ prior to movement and then forcibly stretched through a $70^{\circ}$ arc of plantarflexion at $900^{\circ} / \mathrm{s}$. (a) Trace recordings of torque lengthening contractions (superimposed on a maximal lengthening contraction for $200 \mathrm{~ms}$ ) for repetitions 1, 5, and 10 (black, pink, and red lines, resp.). (b) Maximal isometric torque was recorded at optimal length $\left(L_{0}\right)$ before (black line) and after (red line) injury. Note that $m d x$ muscles generate at least the same absolute force, but they consistently showed a significant drop in torque (yellow arrow) compared to the wild-type muscles, with an average loss of $85 \%$ compared to $32 \%(P<0.01)$ in normal mice. Not only do the $m d x$ muscles sustain more force loss, but this usually occurs very early in the protocol (after the first few, resp.). ${ }^{*}=P<0.05$.

average of $38 \pm 7$ fibers, and more than 45 fields were counted per muscle.

\section{Results}

To induce injury in the tibialis anterior muscle (TA), we superimposed a lengthening contraction onto a maximal isometric contraction (Figure 1(a)). To assess the amount of injury, we measured maximal torque before and after 


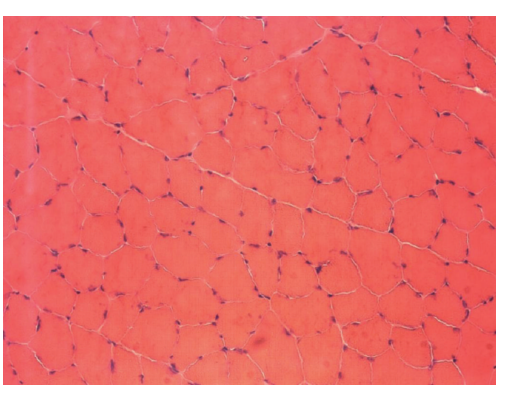

(a)

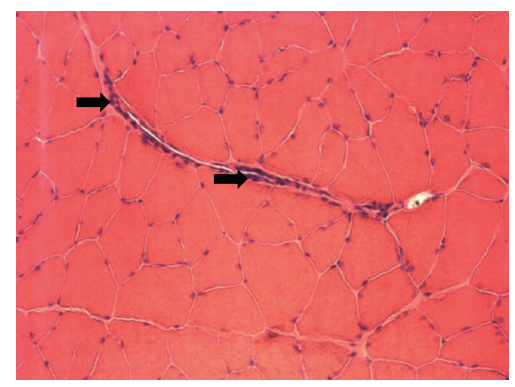

(b)

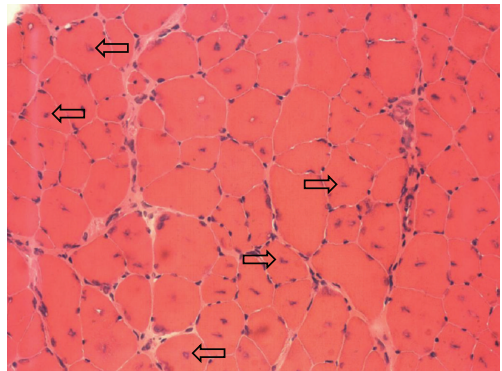

(c)

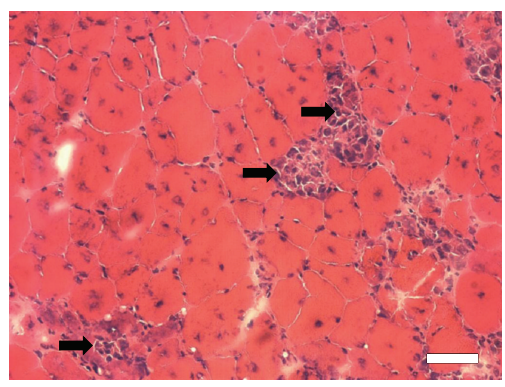

(d)

FIGURE 2: Histopathology related to injury. (a) cross-section of normal healthy TA muscle from a wild-type mouse. Skeletal muscle fibers are multinucleated and the nuclei stain blue; the sarcoplasm of each cell stains pink. (b) cross-section from a wild-type TA after injury. There was only minimal evidence of perivascular inflammation (arrows) in the wild-type tissue after injury. (c) cross-section from TA muscle of an $m d x$ mouse. Even without injury, there is mild inflammation, slight increase in endomysial connective tissue, heterogeneity in fiber size, and many centrally nucleated fibers (CNFs, open arrows), all indicative of ongoing degeneration/regeneration within the muscle. (d) crosssection from an $m d x$ TA after injury. Even with a protocol that produces mild changes in morphology to healthy muscle, the $m d x$ muscle suffers much more damage, such as myonecrosis, myophagocytosis, and foci of inflammation surrounding individual muscle fibers (closed arrows). Scale bar $=40 \mu \mathrm{m}$.

injury (Figure 1(b)). While the mechanism of injury was identical between animals, the injury was much more severe in dystrophic mice, with an average force loss of $85 \%$, compared to $42 \%(P<0.05)$ in normal mice.

Histological changes paralleled functional changes to some extent. For H\&E staining, there was only minimal evidence of perivascular inflammation in the wild-type tissue after injury (Figure 2(b)) compared to undisturbed healthy muscle (Figure 2(a)). Even without injury, centrally nucleated fibers, fiber size variation, mild inflammation, and slight increases in endomysial connective tissue in the uninjured $m d x$ mice are all consistent with ongoing muscle damage and regeneration (Figure 2(c)). In contrast, the myonecrosis, myophagocytosis, and foci of inflammation surrounding individual muscle fibers seen in the injured $m d x$ muscle (Figure 2(d)) are all consistent with acute muscle injury. The amount of membrane damage, as evidenced by the number of fibers with intracellular EBD, also reflected the magnitude of functional injury (Figures $3(\mathrm{a})-3(\mathrm{e})$ ). In wild-type tissue sections (Figure 3(a)), there were few fibers that could be found to have intracellular EBD (1\%); however after the relatively mild injury protocol (Figure $3(\mathrm{~b})$ ), the number of fibers with membrane damage did not increase significantly $(13 \pm 2 \%, P<0.06)$. The uninjured $m d x$ tissue (Figure 3(c)) already had a small amount of EBD-positive fibers $(11 \pm 4 \%, P<0.05)$, but there was almost a 5 -fold increase in the number of fibers with membrane damage after injury $(49 \pm 8 \%, P<0.05$, Figure $3(d))$.

MRI revealed a clear increase on the T2 signal after injury (Figures 4(a) and 4(b)). Quantitative changes in T2 are apparent in Figures 4(c) and 4(d), where the expected $\mathrm{T} 2$ signal was fit across multiple echo times in T2-weighted images [25]. There was no significant difference in parameters between the injured and uninjured leg in the normal mice. Further, there were no significant differences in any of the measured parameters for the TA between normal and dystrophic mice in the uninjured side. However, there were marked differences in parameters in the injured side between normal and dystrophic mice and between the injured and uninjured leg in the dystrophic mice (Table 1). As in prior studies [14], the sham animals did not have an increase in T2 signal by the time imaging was performed.

Figure 5 shows a representative picture of 3D tractography. When comparing parameter of diffusion on the injured side, dystrophic mice showed significantly increased MD and $\mathrm{AD}$ and decreased FA $(P<0.05)$ in the proximal and middle TA compared to wild-type uninjured mice. These differences were much more marked than the more limited differences we measured with T2. While not significant, RD trended toward increased values. These data are plotted against force loss in Figure 6. DTI tractography (see Figure 5, e.g.) was used to visualize the TAs. 


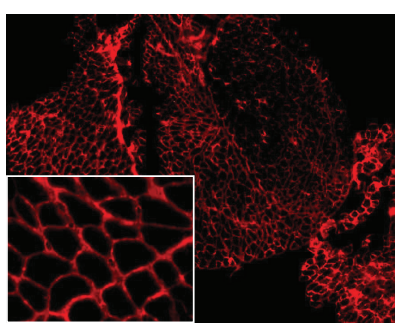

(a)

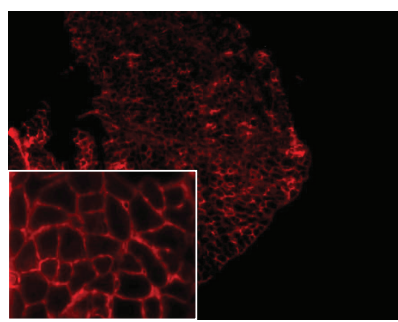

(b)

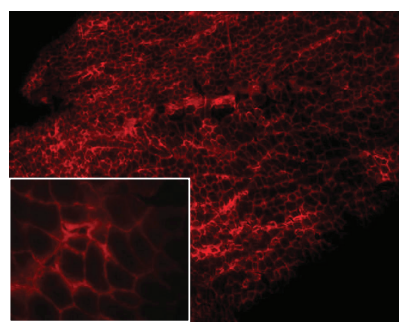

(c)

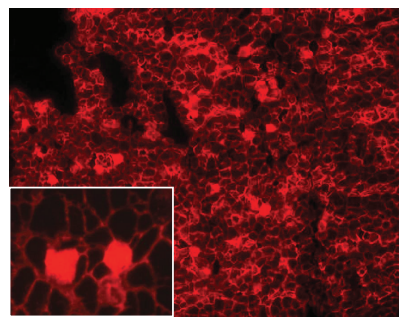

(d)

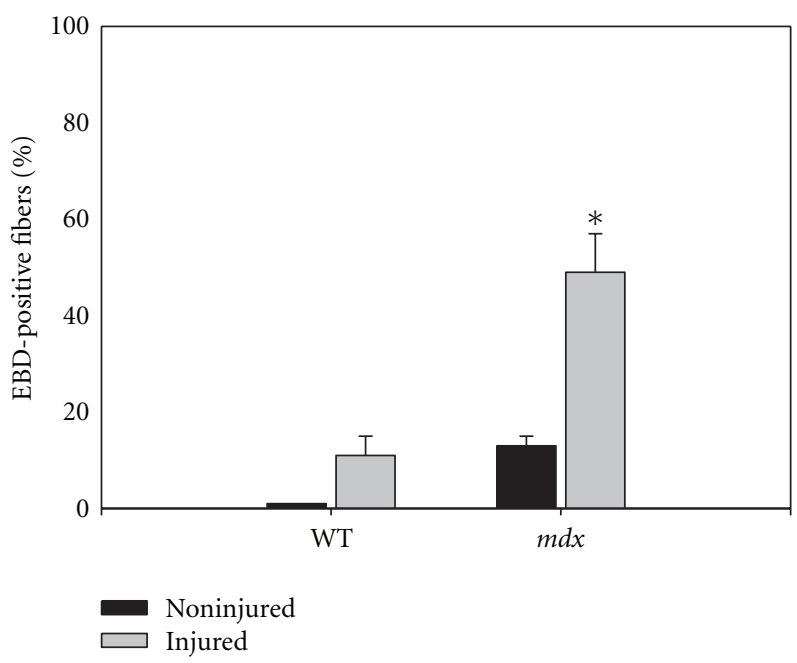

(e)

FIGURE 3: Membrane stability in muscle fibers before and after injury. Evans Blue Dye (EBD) was used to evaluate sarcolemmal integrity within the TA muscles of mice after injury. EBD is detected under fluorescence microscopy (568 nm, insets show high magnification) and the presence of the protein-bound dye inside the muscle fiber indicates damage to the sarcolemma. (a) cryosection of an uninjured wildtype TA muscle from animals injected with EBD. (b) cryosection of an injured wild-type TA; the injury protocol used here was not enough to cause significant membrane damage in wild-type muscle. (c) cryosection of an uninjured $m d x$ TA from animals injected with EBD. (d) cryosection of an injured wild-type TA; intracellular EBD indicates damage to the sarcolemma, which occurred frequently in the $m d x$ injured muscles. (e) Histogram showing quantification of EBD-positive fibers. Without injury, the number of EBD-positive (EBD+) fibers was not significantly different between wild type and $m d x$. Only the $m d x$ animals showed a significant increase in the number of positively labeled fibers after injury. Scale bar $=200 \mu \mathrm{m}$. * : significant difference from noninjured, $P<0.05$.

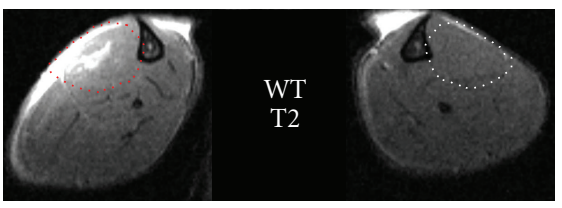

(a)

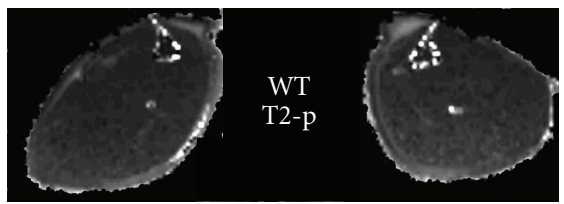

(c)

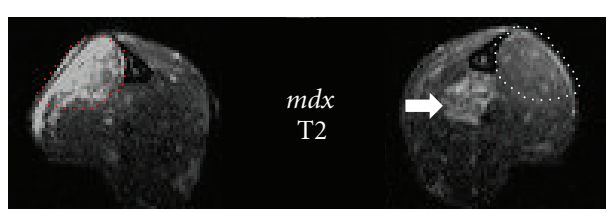

(b)

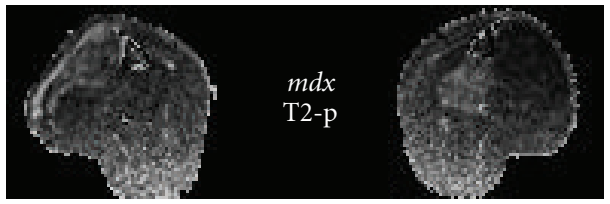

(d)

Figure 4: Representative T2-weighted and T2-parametric images. (a) Example of a T2-weighted image (T2) after injury in the wildtype (WT) mouse. The injured TA (dotted red circle) is easily discerned from the TA on the control side (dotted white circle) based on the increased T2 signal, presumably due to edema. (b) Even in noninjured $m d x$ muscle, there are regions of hyperintensity (arrow), a characteristic finding in dystrophic muscle. (c-d) Example of a T2 parametric image (T2-p) after injury in the wild-type and $m d x$ mouse.

\section{Discussion}

Since X-rays reveal little regarding skeletal muscle anatomy or function, muscle injury is still typically diagnosed by taking a history and performing a physical examination. However, modalities such as MRI are becoming more affordable and more commonplace, and there is a surge of interest in using them to learn more about both muscle injury and muscle disease. Acute muscle strain injury is a stochastic event, and that would not be ethical or desirable to induce in human subjects; therefore most human studies involve imaging after "eccentric exercise" or "exertional muscle injury" (i.e., overuse type injuries). By using an established animal model to induce an acute contractioninduced injury, we were able to compare changes in muscle function, in vivo imaging, and histology shortly after injury. Our injury method clearly resulted in muscle damage, as indicated by the measurable loss of force, changes in $\mathrm{H} \& \mathrm{E}$ staining, and an uptake of EBD by injured muscle fibers, as well as marked changes in MRI parameters, especially DTI. 
TABle 1: Quantitative data between control (wild-type) and dystrophic ( $m d x)$ TA muscles without and with injury. Except for RD, all the calculated water diffusion characteristics were significantly altered $(P<0.05)$ after injury, and to a greater extent in $m d x$ muscles. There was a trend toward increased $\mathrm{RD}$, but this was not significant (NS) at $P<0.05$.

\begin{tabular}{|c|c|c|c|c|}
\hline & $\begin{array}{c}m d x \text { noninjured versus } \\
\text { wt noninjured }\end{array}$ & $\begin{array}{c}\text { wt injured versus } \\
\text { noninjured }\end{array}$ & $\begin{array}{c}m d x \text { injured versus } \\
\text { wt injured }\end{array}$ & $\begin{array}{c}m d x \text { injured versus } \\
\text { noninjured }\end{array}$ \\
\hline Whole MD $\left(\mathrm{mm}^{2} / \mathrm{s}\right)$ & NS & NS & $\begin{array}{c}\uparrow(0.00163 \pm 0.00012 \text { versus } \\
0.00143 \pm 0.000087)\end{array}$ & $\begin{array}{c}\uparrow(0.00163 \pm 0.00012 \\
\text { versus } \\
0.00140 \pm 0.00013)\end{array}$ \\
\hline Proximal MD $\left(\mathrm{mm}^{2} / \mathrm{s}\right)$ & NS & NS & $\begin{array}{c}\uparrow(0.00165 \pm 0.00011 \text { versus } \\
0.00143 \pm 0.00012)\end{array}$ & NS \\
\hline Middle MD $\left(\mathrm{mm}^{2} / \mathrm{s}\right)$ & NS & NS & $\begin{array}{c}\uparrow(0.00161 \pm 0.00013 \text { versus } \\
0.00140 \pm 0.00011)\end{array}$ & NS \\
\hline Distal MD $\left(\mathrm{mm}^{2} / \mathrm{s}\right)$ & NS & NS & NS & NS \\
\hline Whole AD $\left(\mathrm{mm}^{2} / \mathrm{s}\right)$ & NS & NS & $\begin{array}{c}\uparrow(0.00139 \pm 0.00010 \text { versus } \\
0.00118 \pm 0.000056)\end{array}$ & $\begin{array}{c}\uparrow(0.00139 \pm 0.00010 \\
\text { versus } \\
0.00113 \pm 0.000011)\end{array}$ \\
\hline Proximal AD $\left(\mathrm{mm}^{2} / \mathrm{s}\right)$ & NS & NS & $\begin{array}{c}\uparrow(0.00140 \pm 0.000082 \\
\text { versus } 0.00117 \pm 0.000097)\end{array}$ & $\begin{array}{c}\uparrow(0.00140 \pm 0.000082 \\
\text { versus } \\
0.00115 \pm 0.00014)\end{array}$ \\
\hline Middle AD $\left(\mathrm{mm}^{2} / \mathrm{s}\right)$ & NS & NS & $\begin{array}{c}\uparrow(0.00137 \pm 0.00011 \text { versus } \\
0.00116 \pm 0.000076)\end{array}$ & $\begin{array}{c}\uparrow(0.00137 \pm 0.00011 \\
\text { versus } \\
0.00113 \pm 0.00010)\end{array}$ \\
\hline Distal AD $\left(\mathrm{mm}^{2} / \mathrm{s}\right)$ & NS & NS & NS & NS \\
\hline Whole FA & NS & NS & $\begin{array}{c}\uparrow(0.287 \pm 0.013 \text { versus } \\
0.329 \pm 0.031)\end{array}$ & $\begin{array}{c}\uparrow(0.287 \pm 0.013 \\
\text { versus } 0.349 \pm 0.021)\end{array}$ \\
\hline Proximal FA & NS & NS & $\begin{array}{c}\uparrow(0.282 \pm 0.017 \text { versus } \\
0.333 \pm 0.015)\end{array}$ & $\begin{array}{l}\uparrow(0.282 \pm 0.017 \\
\text { versus } 0.348 \pm 0.040)\end{array}$ \\
\hline Middle FA & NS & NS & $\begin{array}{c}\uparrow(0.287 \pm 0.018 \text { versus } \\
0.330 \pm 0.027)\end{array}$ & $\begin{array}{c}\uparrow(0.287 \pm 0.018 \\
\text { versus } 0.341 \pm 0.022)\end{array}$ \\
\hline Distal FA & NS & NS & NS & NS \\
\hline Whole RD $\left(\mathrm{mm}^{2} / \mathrm{s}\right)$ & NS & NS & NS & NS \\
\hline Proximal RD $\left(\mathrm{mm}^{2} / \mathrm{s}\right)$ & NS & NS & NS & NS \\
\hline Middle RD $\left(\mathrm{mm}^{2} / \mathrm{s}\right)$ & NS & NS & NS & NS \\
\hline Distal RD $\left(\mathrm{mm}^{2} / \mathrm{s}\right)$ & NS & NS & NS & NS \\
\hline Whole T2 (ms) & NS & NS & NS & NS \\
\hline Proximal T2 (ms) & NS & NS & $\begin{array}{c}\uparrow(38.93 \pm 2.57 \text { versus } \\
29.93 \pm 5.35)\end{array}$ & NS \\
\hline Middle T2 (ms) & NS & NS & NS & NS \\
\hline Distal T2 (ms) & NS & NS & NS & NS \\
\hline
\end{tabular}

Explanation of DTI parameters (apply to all but the last 4 lines, which report data for T2): MD: mean diffusivity; measure of total diffusion within a voxel; AD: axial diffusivity; diffusion along the longitudinal axis; FA: fractional anisotropy; the fraction of unidirectional diffusion; RD: radial diffusivity; diffusion occurring perpendicular to a track; T2: a time constant describing the exponential decay of signal.

There is a growing number of animal studies that use MRI to assess muscle injury; however, these include such methods as overuse (running downhill), myotoxins, denervation, hindlimb suspension reloading, and ischemiareperfusion $[8,12,26-31]$, rather than by acute muscle strain injury. This can make reproducibility and even relevance to functional outcome difficult to determine. Our in vivo animal model of injury provides several advantages, such as the ability to study exclusively one type of contraction (eccentric), to measure force directly from an individual muscle, and to characterize the biochemical and morphological state of the muscle at defined times following injury and during recovery. Because the animal is anesthetized, we can reliably induce damage to a single muscle group with a known velocity, arc of motion, and contractile level. Effort, such as in a maximal voluntary contraction (MVC), and pain are not confounding factors when assessing changes in function. Downhill treadmill running is an exercise that is sometimes used as an injury model, but there are problems with compliance [32]. The in vivo injury model provides a known dose of injury to a specific muscle group in all animals.

Regardless of the method used to induce damage, the T2 signal in MRI is typically increased significantly after muscle injury, but after some protocols there is a long-term persistence of this signal $[33,34]$, with a time scale that 


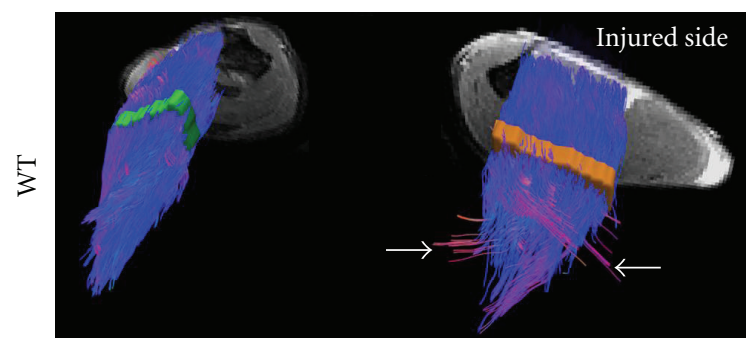

(a)

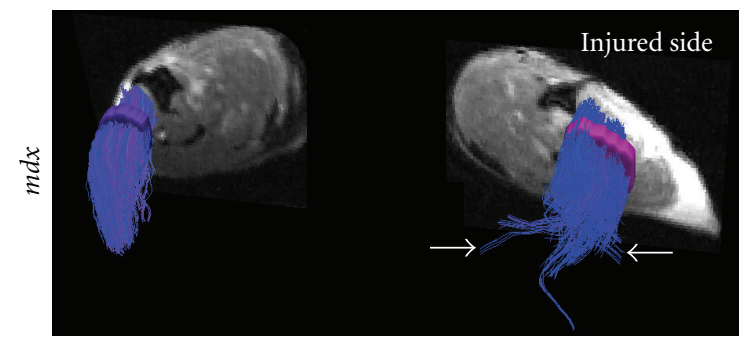

(b)

FIGURE 5: Muscle fiber tracking of the tibialis anterior (TA) muscle in vivo. Examples of "fiber tracking" from processed diffusion tensor imaging (DTI). The image shows modeling of fiber tracks based on the DTI data in the TA muscles of a wild-type (wt) mouse and an $m d x$ mouse in the noninjured and injured sides. Even with this mild injury protocol that shows only minimal change in the T2 signal, the injured TA is readily identified by an apparent interruption in the vertical orientation of the tracks (arrows). Tractography is only a visual depiction of the DTI parameters. It is useful in providing accurate representation of the true anatomy of muscles but less so after injury. The colored bands are just where the regions of interest were drawn to outline the TA. Note that the $m d x$ T2 image shows regions of hyperintensity, as seen in Figure 4.

is not in line with the temporal changes of inflammation and edema [28]. Although some studies have shown that T2 values gradually peak hours or days after injury [26, 30], these employed a myotoxin to injure the muscle. Myotoxins provide a model to study necrosis, inflammation, and massive degeneration and regeneration but do not provide a physiological model of injury. In a recent paper, Mathur et al. [32] used downhill running to study the changes in T2 after eccentric exercise of $m d x$ mice. They used older animals (5-15 months) but still found that $m d x$ mice were more susceptible to damage, with $\mathrm{T} 2$ returning to preexercise levels 10 days after exertion. Interestingly, they noted a strong relationship between the percent of EBD-positive area and the percentage of pixels with elevated T2 $(r=$ 0.79 ). EBD binds to albumin, a blood plasma protein, and is detected by fluorescence microscopy in the extracellular space. Presence of the protein-bound dye inside a muscle fiber indicates damage to the sarcolemma. Since albumin is a relatively large protein $(\sim 67 \mathrm{kDa})$, their findings suggest that downhill running results in relatively large sarcolemmal tears of damaged myofibers.

McIntosh et al. [35] provided one of the first studies to use MRI to assess skeletal muscle in $m d x$ mice. These authors noted heterogeneous signal intensity on T2-weighted images in $m d x$ hindlimb muscles. These foci of high intensity were subsequently confirmed by Walter et al. [36], who used more advanced techniques to obtain higher resolution images as well to quantify the changes in the T2 signal that occurred in the $m d x$ (and $\gamma s g-/-$ ) mice compared to controls. Walter et al. also showed that delivery of $\gamma$ sg by AAV (adenoassociated virus) into hindlimb muscles was not only able to rescue to phenotype (i.e., return of normal histology) but also restored the T2 properties of skeletal muscle.

DTI parameters have been compared to longitudinal sections of the rat TA, the results of which show that DTI directions actually represent the local orientation of fibers in the rat TA muscle [37]. Damon et al. have used DTI fiber tracking successfully to measure pennation angles of myofibers in human skeletal muscle $[10,11,38,39]$, and subsequent studies have also used DTI in mice, both for three-dimensional reconstruction of fiber tracks [40] and as a measure of damage induced by ischemia reperfusion [8]. Heemskerk et al. provide an excellent description of measured changes in DTI parameters after ischemia-reperfusion type of injury in mouse skeletal muscle [12]. They found that DTI indices changed in response to ischemia-reperfusion and that the indices correlated with histopathology, although they caution against overinterpretation since their study was not designed to achieve exact correlation. Such correlation requires optimal MRI protocols, an exact spatial correspondence between histology and MRI slices, and specific immunohistochemical labeling to identify specific cell types.

One can use DTI to compare muscle architecture of dystrophic and healthy hindlimb muscles of mice in vivo. This could be extremely valuable to muscle physiologists who need to obtain the cross-sectional area (CSA) of a muscle to calculate the "specific force" (force normalized to CSA) without removing the muscles. In addition to the computer-generated models of fiber tracks, we were able to collect quantifiable data. Using our injury model, the diffusion parameters (decrease in FA and increases in MD and $\mathrm{AD}$ ) and $\mathrm{T} 2$ findings are consistent with increased edema. However, the lack of significant changes in RD may suggest increased diffusion along the sarcolemma as a result of cell swelling. Interestingly, significant changes in DTI parameters were evident in the middle and proximal sections of the TA, and significant T2 changes were evident in the proximal TA. Such findings support earlier work suggesting that damage to the myofibers is not limited to the muscletendon junction, where soreness often occurs [14].

There are certain limitations to any study of muscle injury and disease. In addition to technical issues involved with imaging [41], position of the limb [42], and the lack of any single biological marker to explain the loss of force after injury $[43,44]$, one must still use caution when interpreting T2 intensity or DTI findings. The exact meaning of T2 changes are still to be elucidated $[28,32$, 45]. While tractography corresponds to normal anatomy, there is a tendency after injury for the more superficial fascicles to appear abnormal (Figure 5, arrows). This threedimensional reconstruction can appear to have fibers that "punch through" the strong connective tissue (epimysium) that surrounds that muscle and enter into the space occupied 


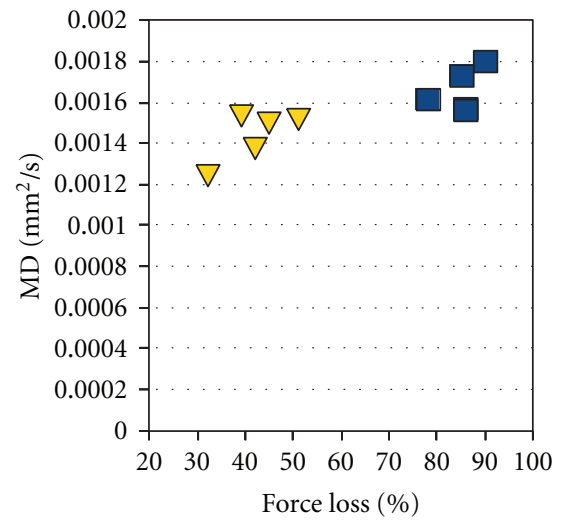

(a)

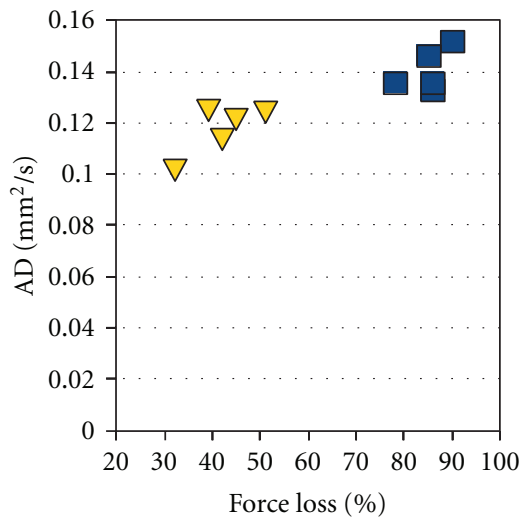

(b)

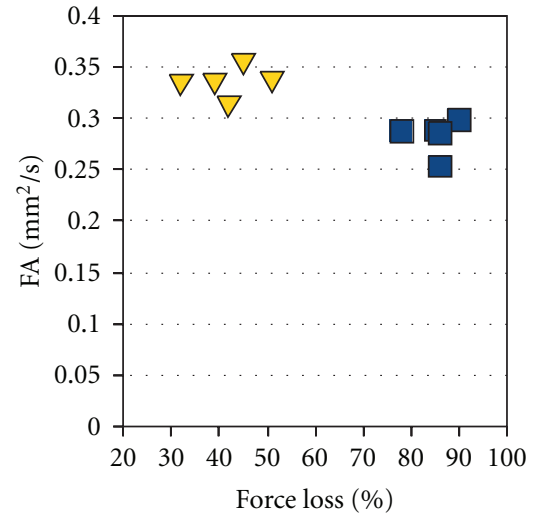

(c)

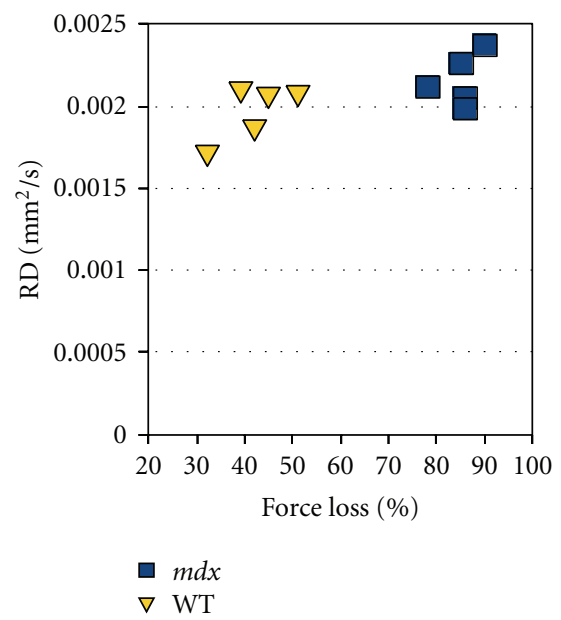

(d)

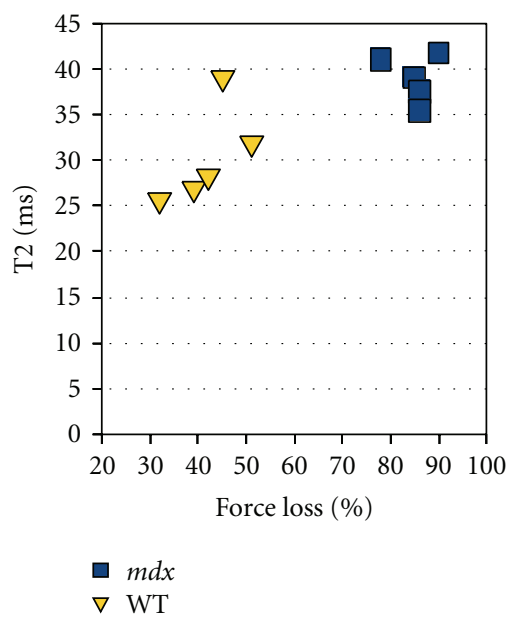

(e)

FIgURE 6: Diffusion tensor imaging (DTI) parameters after injury. Parameters ( $y$-axis, in $\mathrm{mm}^{2} / \mathrm{s}$ ) for the proximal section of TA muscles from wild-type (yellow triangles) and $m d x$ (blue squares) mice are plotted against force loss ( $x$-axis, in \% loss compared to pre-injury torque). Parameters include (a) mean diffusivity (MD), (b) axial diffusivity (AD), (c) fractional anisotropy (FA), (d) radial diffusivity (RD). (e) shows T2 (ms) also presented plotted against force loss. Presenting the data in this way, one can see that as there is a loss in force, there is a greater change in the diffusion parameters for the $m d x$ mice than with the wild-type mice.

by another muscle, when indeed this is not the case. This is likely to be even more of a problem when using DTI on patients with DMD, as they have significant progressive damage over time and a gradual replacement of muscle tissue by fat, something that does not occur in the $m d x$ mouse model of DMD. Such issues are still being worked on and can hopefully be resolved. However, this change in normal direction of myofibers is clearly indicative of damage, and tractography in healthy muscle is still a useful tool to obtain volume in order to calculate "specific force" (force per unit cross-sectional area).

In summary, we have used MRI to study injured muscle by observing edema and muscle fiber diffusion changes with T2 and DTI sequences, respectively. DTI exhibited significant changes in both the middle and proximal sections of the TA, whereas T2 changes were only significant in the proximal section. This is, to the best of our knowledge, the first animal study to use DTI in a reliable and valid animal model of eccentric injury. The results suggest that DTI is a reliable marker in the assessment of acute muscle injury, even at early time points where the MR signal changes are dominated by local edema.

\section{Acknowledgment}

This work was supported by Grants to RML from the National Institutes of Health (K01AR053235 and 1R01AR059179).

\section{References}

[1] G. Duchenne, De l'électrisation localisée et de son application a la pathologie et a la thérapeutique, Bailliere et Fils, Paris, France, 1861.

[2] W. E. Garrett Jr., "Muscle strain injuries," American Journal of Sports Medicine, vol. 24, pp. S2-S8, 1996.

[3] K. D. Hunter and J. A. Faulkner, "Pliometric contractioninduced injury of mouse skeletal muscle: effect of initial 
length," Journal of Applied Physiology, vol. 82, no. 1, pp. 278283, 1997.

[4] C. Dellorusso, R. W. Crawford, J. S. Chamberlain, and S. V. Brooks, "Tibialis anterior muscles in $\mathrm{mdx}$ mice are highly susceptible to contraction-induced injury," Journal of Muscle Research and Cell Motility, vol. 22, no. 5, pp. 467-475, 2001.

[5] B. J. Petrof, J. B. Shrager, H. H. Stedman, A. M. Kelly, and H. L. Sweeney, "Dystrophin protects the sarcolemma from stresses developed during muscle contraction," Proceedings of the National Academy of Sciences of the United States of America, vol. 90, no. 8, pp. 3710-3714, 1993.

[6] K. P. Speer, J. Lohnes, W. E. Garrett Jr., and E. Eriksson, "Radiographic imaging of muscle strain injury," American Journal of Sports Medicine, vol. 21, no. 1, pp. 89-96, 1993.

[7] D. G. Blankenbaker and A. A. De Smet, "MR imaging of muscle injuries," Applied Radiology, vol. 33, no. 4, pp. 14-26, 2004.

[8] A. M. Heemskerk, M. R. Drost, G. S. Van Bochove, M. F. M. Van Oosterhout, K. Nicolay, and G. J. Strijkers, "DTI-based assessment of ischemia-reperfusion in mouse skeletal muscle," Magnetic Resonance in Medicine, vol. 56, no. 2, pp. 272-281, 2006.

[9] C. H. Sotak, "The role of diffusion tensor imaging in the evaluation of ischemic brain-a review," NMR in Biomedicine, vol. 15, no. 7-8, pp. 561-569, 2002.

[10] B. M. Damon, Z. Ding, A. W. Anderson, A. S. Freyer, and J. C. Gore, "Validation of diffusion tensor MRI-based muscle fiber tracking," Magnetic Resonance in Medicine, vol. 48, no. 1, pp. 97-104, 2002

[11] A. M. Heemskerk, T. K. Sinha, K. J. Wilson, Z. Ding, and B. M. Damon, "Quantitative assessment of DTI-based muscle fiber tracking and optimal tracking parameters," Magnetic Resonance in Medicine, vol. 61, no. 2, pp. 467-472, 2009.

[12] A. M. Heemskerk, G. J. Strijkers, M. R. Drost, G. S. Van Bochove, and K. Nicolay, "Skeletal muscle degeneration and regeneration after femoral artery ligation in mice: monitoring with diffusion MR imaging," Radiology, vol. 243, no. 2, pp. 413-421, 2007.

[13] R. M. Lovering, A. O’Neill, J. M. Muriel, B. L. Prosser, J. Strong, and R. J. Bloch, "Physiology, structure, and susceptibility to injury of skeletal muscle in mice lacking keratin 19-based and desmin-based intermediate filaments," American Journal of Physiology, vol. 300, no. 4, pp. C803-C813, 2011.

[14] R. M. Lovering, A. B. Mcmillan, and R. P. Gullapalli, "Location of myofiber damage in skeletal muscle after lengthening contractions," Muscle and Nerve, vol. 40, no. 4, pp. 589-594, 2009.

[15] R. M. Lovering, J. A. Roche, R. J. Bloch, and P. G. De Deyne, "Recovery of function in skeletal muscle following 2 different contraction-induced injuries," Archives of Physical Medicine and Rehabilitation, vol. 88, no. 5, pp. 617-625, 2007.

[16] R. M. Lovering and P. G. De Deyne, "Contractile function, muscle eccentric contraction-induced injury," American Journal of Physiology, vol. 286, no. 2, pp. C230-C238, 2004.

[17] I. A. Barash, L. Mathew, A. F. Ryan, J. Chen, and R. L. Lieber, "Rapid muscle-specific gene expression changes after a single bout of eccentric contractions in the mouse," American Journal of Physiology, vol. 286, no. 2, pp. C355-C364, 2004.

[18] C. P. Ingalls, G. L. Warren, J. Z. Zhang, S. L. Hamilton, and R. B. Armstrong, "Dihydropyridine and ryanodine receptor binding after eccentric contractions in mouse skeletal muscle," Journal of Applied Physiology, vol. 96, no. 5, pp. 1619-1625, 2004.
[19] M. Hakim, W. Hage, R. M. Lovering, C. T. Moorman, L. A. Curl, and P. G. De Deyne, "Dexamethasone and recovery of contractile tension after a muscle injury," Clinical Orthopaedics and Related Research, no. 439, pp. 235-242, 2005.

[20] R. M. Lovering, M. Hakim, C. T. Moorman, and P. G. De Deyne, "The contribution of contractile pre-activation to loss of function after a single lengthening contraction," Journal of Biomechanics, vol. 38, no. 7, pp. 1501-1507, 2005.

[21] R. M. Lovering, J. A. Roche, M. H. Goodall, B. B. Clark, and A. Mcmillan, "An in vivo rodent model of contraction-induced injury and non-invasive monitoring of recovery," Journal of Visualized Experiments, no. 51, 2011.

[22] S. Mori, B. J. Crain, V. P. Chacko, and P. C. M. Van Zijl, “Threedimensional tracking of axonal projections in the brain by magnetic resonance imaging," Annals of Neurology, vol. 45, no. 2, pp. 265-269, 1999.

[23] P. W. Hamer, J. M. McGeachie, M. J. Davies, and M. D. Grounds, "Evans Blue Dye as an in vivo marker of myofibre damage: optimising parameters for detecting initial myofibre membrane permeability," Journal of Anatomy, vol. 200, no. 1, pp. 69-79, 2002.

[24] M. R. Stone, A. O’Neill, R. M. Lovering et al., “Absence of keratin 19 in mice causes skeletal myopathy with mitochondrial and sarcolemmal reorganization," Journal of Cell Science, vol. 120, no. 22, pp. 3999-4008, 2007.

[25] H. K. Kim, T. Laor, P. S. Horn, J. M. Racadio, B. Wong, and B. J. Dardzinski, "T2 mapping in Duchenne muscular dystrophy: distribution of disease activity and correlation with clinical assessments," Radiology, vol. 255, no. 3, pp. 899-908, 2010.

[26] K. T. Mattila, R. Lukka, T. Hurme, M. Komu, A. Alanen, and H. Kalimo, "Magnetic resonance imaging and magnetization transfer in experimental myonecrosis in the rat," Magnetic Resonance in Medicine, vol. 33, no. 2, pp. 185-192, 1995.

[27] C. Sébrié, B. Gillet, J. P. Lefaucheur, A. Sébille, and J. C. Beloeil, "Mouse muscle regeneration: an in vivo 2D $1 \mathrm{H}$ magnetic resonance spectroscopy (MRS) study," FEBS Letters, vol. 423, no. 1, pp. 71-74, 1998.

[28] T. Marqueste, B. Giannesini, Y. Le Fur, P. J. Cozzone, and D. Bendahan, "Comparative MRI analysis of T2 changes associated with single and repeated bouts of downhill running leading to eccentric-induced muscle damage," Journal of Applied Physiology, vol. 105, no. 1, pp. 299-307, 2008.

[29] J. Zhang, G. Zhang, B. Morrison, S. Mori, and K. A. Sheikh, "Magnetic resonance imaging of mouse skeletal muscle to measure denervation atrophy," Experimental Neurology, vol. 212, no. 2, pp. 448-457, 2008.

[30] A. Wishnia, H. Alameddine, S. Tardif de Géry, and A. LeroyWillig, "Use of magnetic resonance imaging for noninvasive characterization and follow-up of an experimental injury to normal mouse muscles," Neuromuscular Disorders, vol. 11, no. 1 , pp. 50-55, 2001.

[31] T. N. Frimel, G. A. Walter, J. D. Gibbs, G. S. Gaidosh, and $\mathrm{K}$. Vandenborne, "Noninvasive monitoring of muscle damage during reloading following limb disuse," Muscle and Nerve, vol. 32, no. 5, pp. 605-612, 2005.

[32] S. Mathur, R. S. Vohra, S. A. Germain et al., "Changes in muscle T2 and tissue damage after downhill running in mdx mice," Muscle and Nerve, vol. 43, no. 6, pp. 878-886, 2011.

[33] J. L. Fleckenstein, P. T. Weatherall, R. W. Parkey, J. Payne, and R. M. Peschock, "Sports-related muscle injuries: evaluation with MR imaging," Radiology, vol. 172, no. 3, pp. 793-798, 1989.

[34] F. G. Shellock, T. Fukunaga, J. H. Mink, and V. R. Edgerton, "Exertional muscle injury: evaluation of concentric versus 
eccentric actions with serial MR imaging," Radiology, vol. 179, no. 3, pp. 659-664, 1991.

[35] L. M. McIntosh, R. E. Baker, and J. E. Anderson, "Magnetic resonance imaging of regenerating and dystrophic mouse muscle," Biochemistry and Cell Biology, vol. 76, no. 2-3, pp. 532-541, 1998.

[36] G. Walter, L. Cordier, D. Bloy, and H. L. Sweeney, "Noninvasive monitoring of gene correction in dystrophic muscle," Magnetic Resonance in Medicine, vol. 54, no. 6, pp. 1369-1376, 2005.

[37] C. C. Van Donkelaar, L. J. G. Kretzers, P. H. M. Bovendeerd et al., "Diffusion tensor imaging in biomechanical studies of skeletal muscle function," Journal of Anatomy, vol. 194, no. 1, pp. 79-88, 1999.

[38] J. H. Kan, A. M. Heemskerk, Z. Ding et al., "DTI-based muscle fiber tracking of the quadriceps mechanism in lateral patellar dislocation," Journal of Magnetic Resonance Imaging, vol. 29, no. 3, pp. 663-670, 2009.

[39] D. A. Lansdown, Z. Ding, M. Wadington, J. L. Hornberger, and B. M. Damon, "Quantitative diffusion tensor MRI-based fiber tracking of human skeletal muscle," Journal of Applied Physiology, vol. 103, no. 2, pp. 673-681, 2007.

[40] A. M. Heemskerk, G. J. Strijkers, A. Vilanova, M. R. Drost, and K. Nicolay, "Determination of mouse skeletal muscle architecture using three-dimensional diffusion tensor imaging," Magnetic Resonance in Medicine, vol. 53, no. 6, pp. 1333-1340, 2005.

[41] B. M. Damon, "Effects of image noise in muscle diffusion tensor (DT)-MRI assessed using numerical simulations," Magnetic Resonance in Medicine, vol. 60, no. 4, pp. 934-944, 2008.

[42] U. Sinha, S. Sinha, J. A. Hodgson, and R. V. Edgerton, "Human soleus muscle architecture at different ankle joint angles from magnetic resonance diffusion tensor imaging," Journal of Applied Physiology, vol. 110, no. 3, pp. 807-819, 2011.

[43] G. L. Warren, C. P. Ingalls, D. A. Lowe, and R. B. Armstrong, "What mechanisms contribute to the strength loss that occurs during and in the recovery from skeletal muscle injury?" Journal of Orthopaedic and Sports Physical Therapy, vol. 32, no. 2, pp. 58-64, 2002.

[44] D. L. Morgan and D. G. Allen, "Early events in stretch-induced muscle damage," Journal of Applied Physiology, vol. 87, no. 6, pp. 2007-2015, 1999.

[45] R. A. Meyer and B. M. Prior, "Functional magnetic resonance imaging of muscle," Exercise and Sport Sciences Reviews, vol. 28, no. 2, pp. 89-92, 2000. 

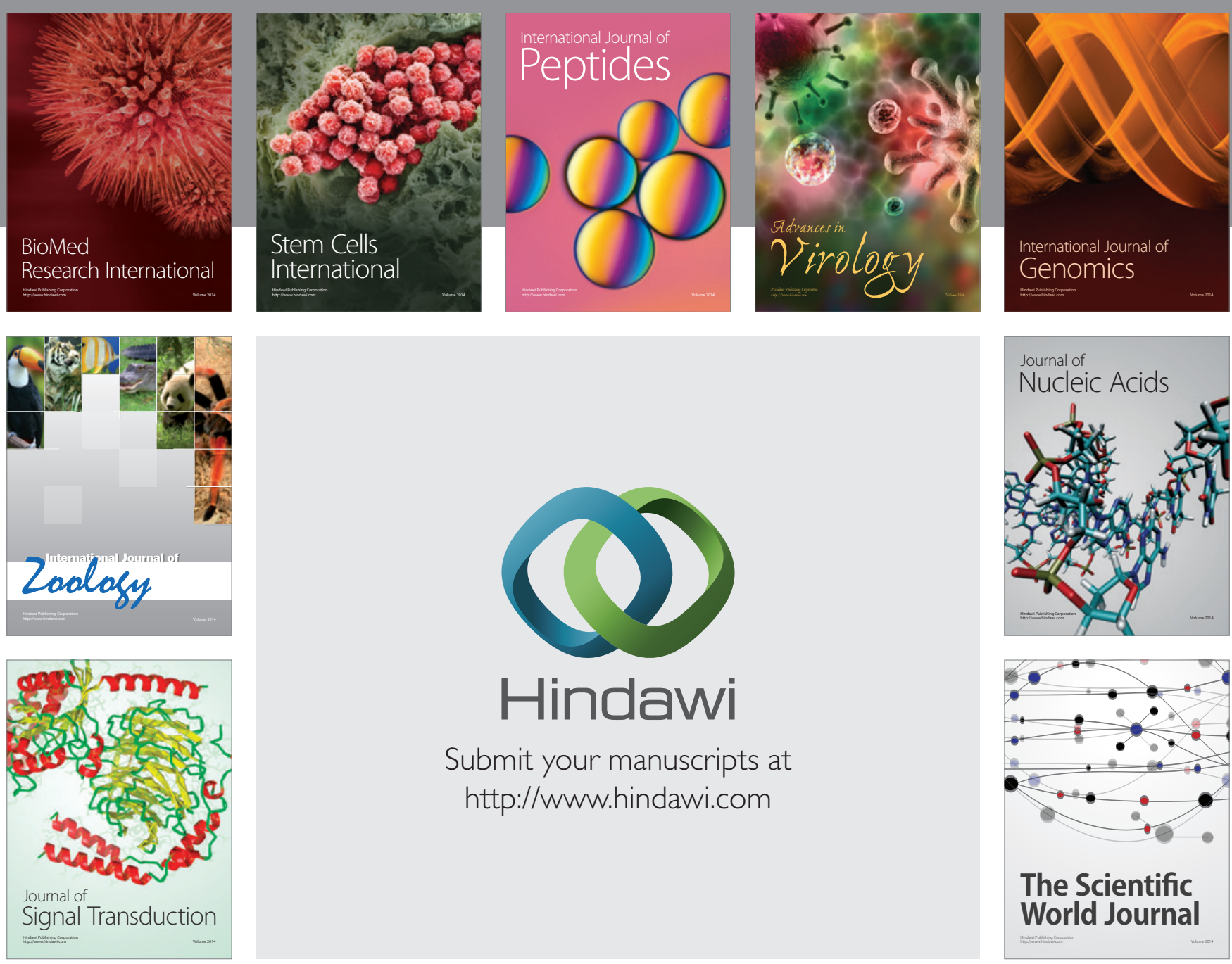

Submit your manuscripts at

http://www.hindawi.com
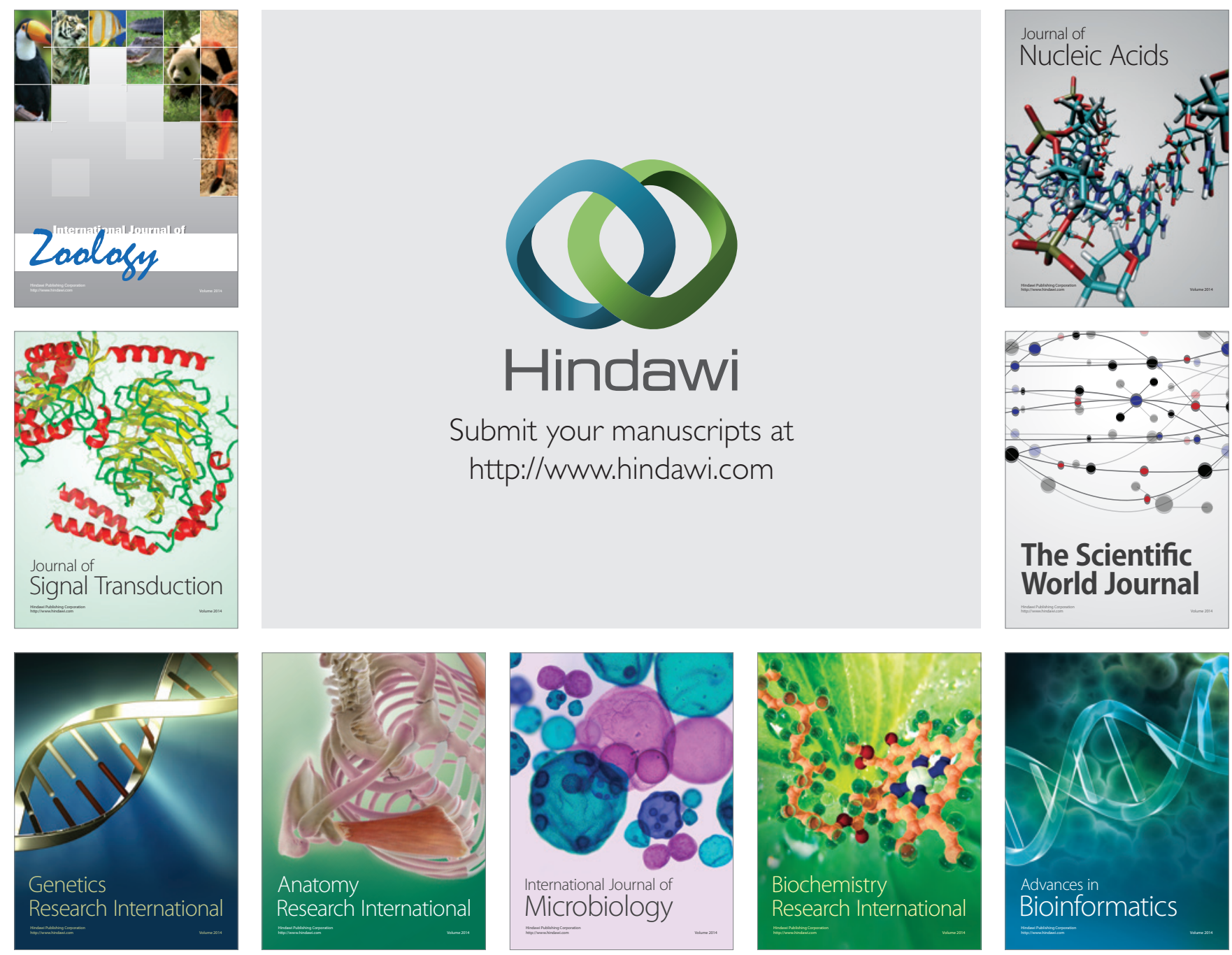

The Scientific World Journal
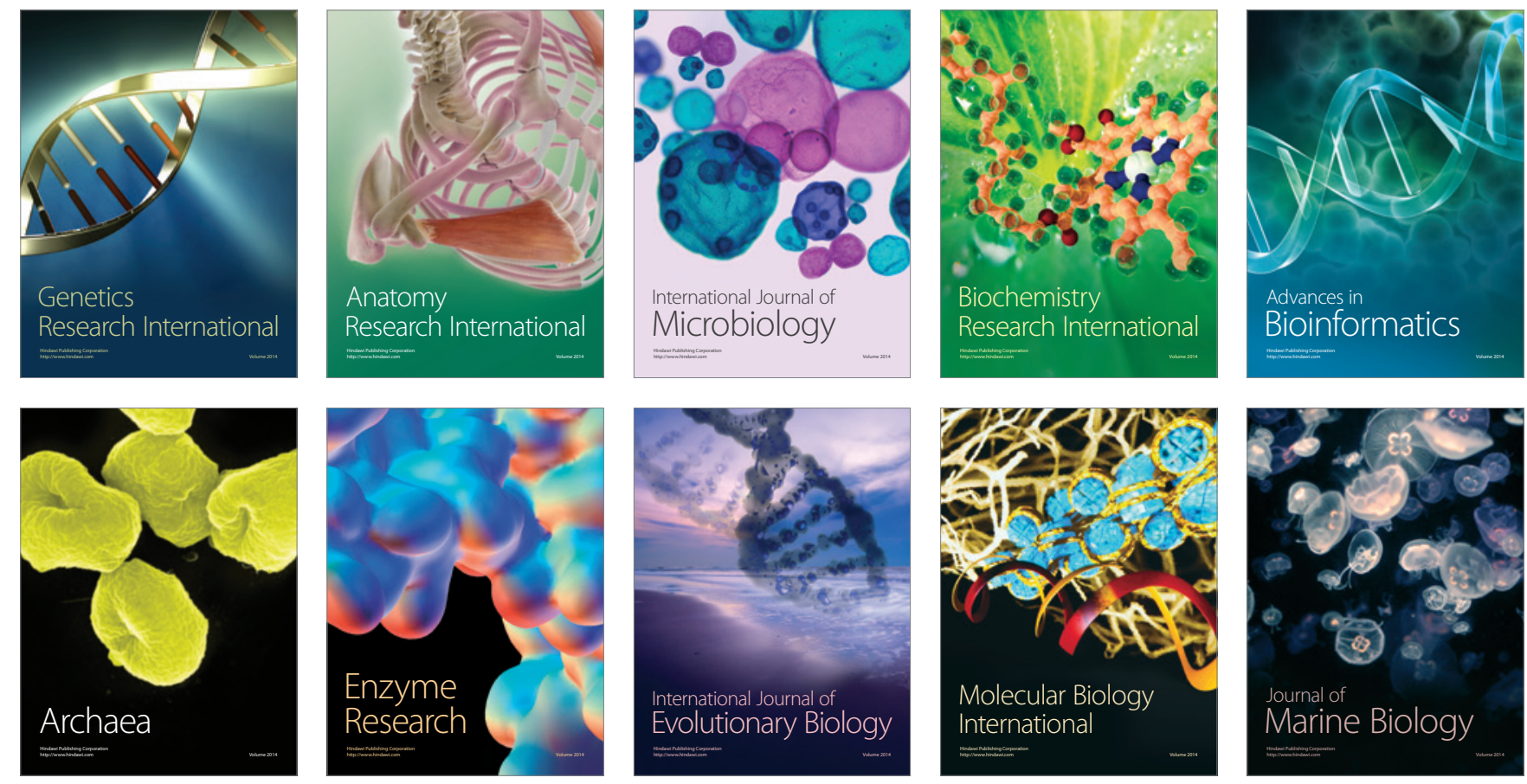